\title{
Coulisses
}

Revue de théâtre

10 | Printemps 1994

Varia

\section{A la recherche du désir perdu : Der Park de Botho Strauss}

Gérard Jugan

\section{(2) OpenEdition}

1 Journals

Édition électronique

URL : http://journals.openedition.org/coulisses/3011

DOI : 10.4000/coulisses.3011

ISSN : 2546-9460

Éditeur

Presses universitaires de Franche-Comté

\section{Édition imprimée}

Date de publication : 1 juin 1994

Pagination : 52-59

ISSN : 1150-594X

\section{Référence électronique}

Gérard Jugan, «A la recherche du désir perdu : Der Park de Botho Strauss », Coulisses [En ligne], 10 |

Printemps 1994, mis en ligne le 15 mars 2019, consulté le 22 octobre 2019. URL : http://

journals.openedition.org/coulisses/3011 ; DOI : 10.4000/coulisses.3011

Ce document a été généré automatiquement le 22 octobre 2019.

Coulisses 


\title{
A la recherche du désir perdu ${ }^{1}:$ Der Park de Botho Strauss
}

\author{
Gérard Jugan
}

Le Parc à Berlin

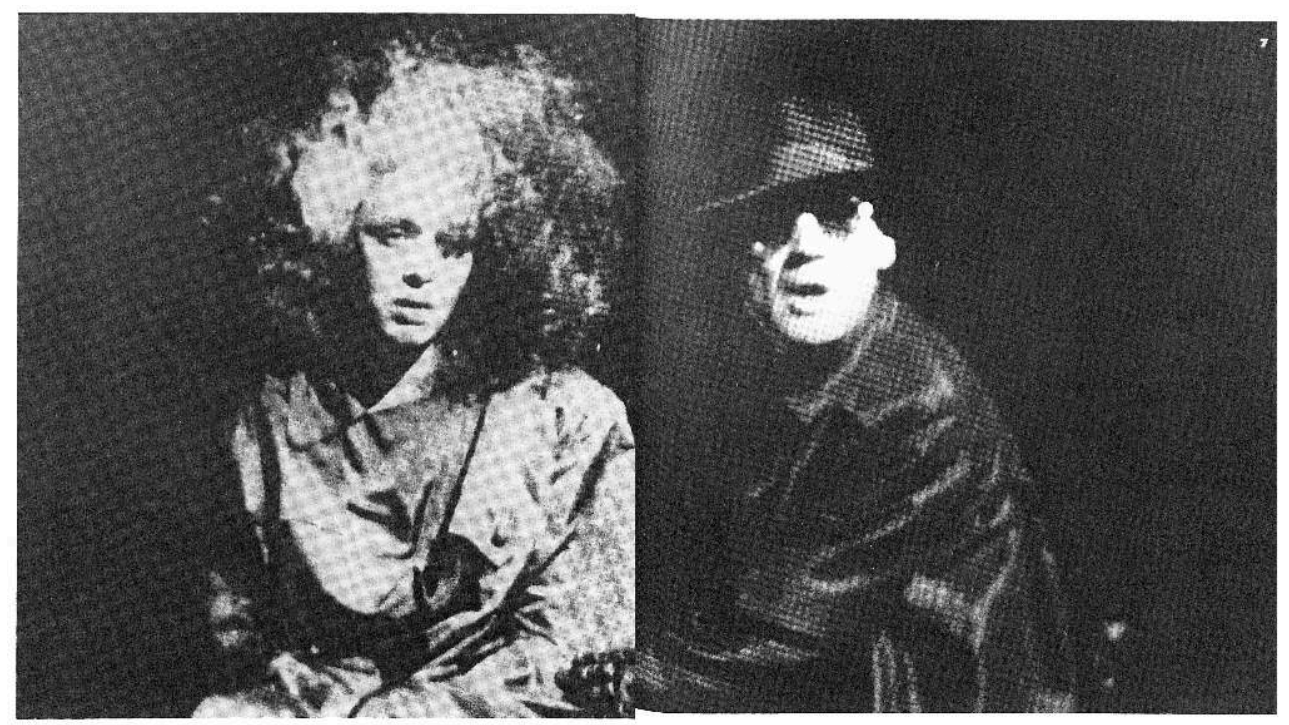

Acte I, scène 3 : Titania (Jutta Lampe) et Oberon (Bruno Ganz), deux divinités envoyées sur terre pour réapprendre le désir aux hommes

1 Der Park est la sixième pièce de Botho Strauss. Elle a constitué sans conteste l'événement de la saison 1984-85 en Allemagne fédérale. Peter Stein, à qui il l'a dédiée, devait être le premier à la monter à la Schaubühne de Berlin, mais l'accident survenu à un acteur au cours d'une répétition l'obligea à différer la date de la première, qui eut lieu le 4 novembre 1984. Entre-temps, Fribourg et Munich l'avaient déjà jouée et une vingtaine de scènes, inscrite au programme de leur saison théâtrale. Depuis le succès de Gross und klein et de Kalldewey, Farce, Botho Strauss remplit les salles de spectacles, même s'il représente parfois pour la critique littéraire une pomme de discorde. 
2 Der Park est une pièce ambitieuse et d'un accès sans doute plus malaisé que les précédentes. Strauss, fidèle à sa démarche, s'y livre à une critique sévère de la société ouest-allemande, dont il est un observateur particulièrement sensible et sans complaisance. Les conclusions de Der Park rejoignent celle de ses autres œuvres; elles dressent à l'évidence un constat de faillite et un lecteur familier de cet auteur y retrouvera sans peine les thèmes favoris d'une vision plutôt sombre de la vie sociale et de l'existence en général : celui de la dérive, de la désintégration de notre société industrielle qui s'illusionne sur sa santé et sa pérennité. Par contre, un lecteur ou un spectateur moins averti restera sans doute assez déconcerté en face de cette œuvre subtile, complexe, mais aussi très longue - une représentation dure plus de cinq heures - et richement chargée de références culturelles. En effet, Strauss, développant une tendance que l'on peut déjà constater dans ses œuvres antérieures, y fait intervenir le fantastique en mobilisant cette fois-ci la mythologie : celle que draine Le Songe d'une nuit d'été dont Der Park est explicitement, non pas une paraphrase mais plutôt une variation désenchantée, au sens propre du terme, ainsi que la mythologie grecque, avec des allusions à des personnages tels que ceux de Pasiphaé, du Minotaure, de Dédale et de Daphné. Cet amalgame n'a en soi rien de choquant; il procède du même artifice que l'on trouve aux sources de toute mythologie et dont le théâtre a déjà fait un large usage : organiser la rencontre des Dieux et des Hommes, pour montrer généralement la carence des uns ou des autres. L'idée poétique de la pièce, à cet égard, est claire : le recours au mythe a pour objectif de médiatiser la critique sociale. Les divinités de Shakespeare et les héros archaïques, redescendus sur terre, en l'occurrence dans un parc d'une métropole moderne (Le Tiergarten de Berlin par exemple), se proposent de mesurer la route parcourue par les humains et de les ramener dans le droit chemin de la vie originelle, dévoyée par les méfaits de la civilisation. Une scène de la pièce nous montre Titania, la reine des fées, captive, dans le parc, des mailles d'un filet qu'ont jeté sur elle une bande de jeunes gens qui la gardent prisonnière, comme un animal. Cette scène est à l'image de Der Park: Oberon et Titania, revenus pour tenter de redonner aux êtres humains ce qu'ils n'ont plus et qui faisait d'eux, autrefois, des hommes: le désir, vont être pris au piège d'une société, aliénée elle aussi, qui va en quelque sorte les absorber sans rémission possible. Botho Strauss utilise, pour montrer cette assimilation réductrice, le cadre dramatique de l'œuvre de Shakespeare. Essayons tout d'abord, pour mieux mettre en lumière la pente de cette pièce, de discerner, parmi les personnages et les situations qu'il nous présente, ceux qu'il emprunte manifestement à son modèle.

\section{Der Park et Le Songe d'une nuit d'été}

3 Strauss laisse dans l'ombre le couple de Thésée et d'Hippolyte pour ne retenir que les personnages majeurs qui articulent véritablement l'action : Titania et Oberon, la reine des fées et le roi des elfes, secondé dans ses entreprises par Cyprian, artiste sculpteur, à qui il délègue des pouvoirs magiques; Cyprian, résurgence du Puck de Shakespeare mais dont Strauss va faire le témoin d'une réflexion sur le statut de l'Art et de l'artiste dans la société moderne. Face à eux, les deux couples humains dont les appartements successifs, sous l'effet de la magie, vont constituer, comme dans Le Songe d'une nuit d'été, le corps dramatique de la pièce. Non plus Lyssandre et Hermia, mais Georg, avocat défenseur des marginaux de la société et Helen, trapéziste dans un cirque, 
d'origine américaine. Non plus Demetrius et Helena mais Wolf, ancien étudiant en histoire, successeur de son père à la tête d'une auto-école, mari de Helma. Quant aux autres personnages, la référence à Shakespeare est plus floue. Le couple des deux amis homosexuels Hofling et Erstling, représentants également de la classe moyenne aisée l'un est architecte, l'autre publicitaire - est-il une variante ironique des artisansacteurs qui jouent la tragédie de Pyrame et Thisbée ? Ce sont effectivement de piètres acteurs, interprètes d'une pièce médiocre, comme le donne à penser le bavardage incontinent et décousu qui marque leur dialogue. Hofling, Erstling: ces noms cherchent-ils à évoquer, dans une tonalité bouffonne, le carriérisme et la courtisanerie chez ces artisans de la société moderne? Leur couple forme un contraste très violent avec le groupe des quatre jeunes gens, trois garçons et une fille, dont le parc est le territoire d'élection. Ils incarnent face aux deux autres couples établis, logés dans des appartements très chics et confortables, avec "vue sur le parc ", une jeunesse démunie, désorientée et révoltée, en rupture avec le monde des adultes qu'elle agresse volontiers. Peut-être faut-il voir en eux, comme le fait Peter Stein, les descendants des elfes et des fées de Shakespeare, chargés comme eux de semer un désordre salutaire, au même titre que Puck, empêcheur de s'assoupir en rond. Le personnage muet du jeune noir, balayeur dans le parc, évoque celui du prince indien, objet dans Le Songe d'une nuit d'été de la rivalité amoureuse d'Oberon et Titania mais que se disputent, chez Strauss, Titania et Cyprian. Tous ces personnages sont reliés entre eux par le parc où ils se rencontrent. Le parc, forme moderne, dégradée, de la forêt athénienne, lieu magique de l'enchantement chez Shakespeare, espace du désenchantement chez Strauss, comme on peut le constater en comparant l'action des deux pièces.

4 Le premier acte a plus d'ampleur que dans Le Songe d'une nuit d'été. Strauss noue d'emblée tous les fils qui vont commander l'action. La liaison entre Georg et Helen s'ébauche et du même coup le ménage à trois que Georg souhaite instaurer avec Wolf, son meilleur ami. Les «témoins » du parc entrent en scène, Hofling et Erstling ainsi que la bande des quatre jeunes gens. Oberon et Titania exposent le but de leur " mission » et se plaignent d'avoir échoué jusqu'à présent dans leurs tentatives exhibitionnistes, restées sans échos. Il s'agit d'un acte d'exposition très dense, au rythme rapide. Les différentes familles de personnages et la trame de leurs relations sont clairement présentées. Le second acte est placé dans les deux pièces sous le signe du sortilège. Strauss expose les prémisses de l'envoûtement dont va naître la crise du troisième acte. Wolf achète à Cyprian une figurine magique pour l'offrir à Helen en cadeau de mariage et s'attirer ses faveurs. Oberon et Cyprian passent au cou de Titania une amulette destinée à calmer son ardeur amoureuse, ce qui a pour effet de la plonger dans une sorte de léthargie et de la transformer en un personnage de "femme d'une autre époque ", léthargie mise à profit par les jeunes gens du parc pour agresser la reine des fées. L'acte se clôt par une discussion entre Wolf et Helma. Elle reproche à son mari l'assoupissement de leur vie conjugale et le tarissement de sa curiosité intellectuelle. Botho Strauss suit toujours Shakespeare au troisième acte, dans les deux pièces, l'acte de la nuit et de la confusion, où le désordre règne en maître et menace dangereusement tous les personnages, hommes et dieux : folie de Titania dans Le Songe d'une nuit d'été, amante éperdue de l'homme fruste à la tête d'âne, folie meurtrière des deux hommes épris de la femme délaissée jusqu'alors. Folie également ou plutôt violence exacerbée chez les personnages de Strauss : violence verbale de Helen qui tient brusquement des propos d'un racisme virulent, violence de Erstling qui tente dans le parc d'abuser de la jeune fille du groupe des jeunes gens, violence de ces mêmes jeunes gens qui capturent 
Titania et la gardent prisonnière, violence extrême du désir sexuel de Titania-Pasiphaé implorant Cyprian-Dédale de lui confectionner le corps d'une vache afin qu'elle puisse s'accoupler à un taureau, violence du désir de Georg pour Helma : elle a, elle aussi, acheté à Cyprian une figurine magique pour reconquérir l'amour de son mari, devenu l'amant d'Helma. C'est à la fin du troisième acte que Strauss amorce la rupture avec $\boldsymbol{L} \boldsymbol{e}$ Songe d'une nuit d'été où Oberon reste maître du jeu et parvient, avec l'aide de Puck, après avoir mené les personnages au bord du chaos, à imposer l'équilibre et l'harmonie. L'Oberon de Botho Strauss avoue son impuissance à mener à bien son entreprise. Il se révoque lui-même, congédie son serviteur et annonce son intention de devenir semblable à ces humains, que la magie n'a pas réussi à éveiller au « désir subtil » qu'il voulait susciter en eux. Les amulettes de Cyprian n'ont pas eu l'effet salutaire escompté, elles ont provoqué des effets négatifs, un dérapage, preuves de son incapacité à transformer des êtres victimes d'une époque dont l'action délétère pervertit le désir des hommes. Les deux derniers actes représentent chez Shakespeare le retour à l'ordre et la fête. Titania reprend ses esprits, les amants se réveillent, retrouvent l'être aimé et les artisans peuvent commencer à répéter la pièce qui va conclure au cinquième acte, dans une tonalité joyeuse, poétique et comique à la fois, $L e$ Songe d'une nuit d'été. Cette détente heureuse, après l'angoisse de la nuit et ses aberrations est totalement absente de la pièce de Strauss qui prolonge, tout au contraire, la plongée amorcée au troisième acte dans le vide et l'obscurité. Ainsi que le souligne fort justement Peter Stein, la courbe dramatique est fondamentalement différente de celle de la pièce de Shakespeare dans la mesure où elle ne se conclut pas par un rétablissement, une résolution finale des conflits, mais présente, de manière linéaire, une dégradation constante et irréversible. Les deux derniers actes de Der Park sont ceux de l'enlisement et de l'absence. Helma retrouve Wolf, mais un Wolf confiné dans un conservatisme petit-bourgeois. Helen, repoussée par les deux hommes, se réfugie dans un appartement où elle a une liaison avec la Mort, expression symbolique d'un désir suicidaire. Cyprian est assassiné dans le parc par le jeune noir. Oberon devenu Oberon / Mittentzwei - l'homme du milieu - a été engagé comme chef des ventes dans l'agence de publicité (elle « vend » des voyages par vidéo-cassettes) qu'ont fondée ensemble Georg et Hôfling; il a troqué son manteau de roi des elfes contre un complet-veston et désappris le langage poétique de Shakespeare. Deux actes placés sous le signe du vide, de la mort, de l'effacement de la personnalité. Botho Strauss y suggère l'image d'une vie sociale où les êtres sont interchangeables, sans visage, une image en négatif du dernier acte du Songe d'une nuit d'été, fête du spectacle et de la joie de vivre avec Shakespeare qui donne à contrario une leçon de bon théâtre (et de vie authentique) en dénonçant la pesanteur des clichés et des conventions. Théâtre de la banalité la plus exemplaire, du nivellement des êtres, de leurs relations et de leur langage chez Strauss. Oberon / Mittentzwei a eu un accident de la circulation avec la voiture de Helen et Georg et tous trois discutent dans un café des modalités du constat. Titania retrouve Oberon, dissimulé derrière $\mathrm{Ob} / \mathrm{Mit}$, ainsi que le nomme Strauss à l'acte IV, mais Oberon reste sourd aux vers de Shakespeare. Pyrame et Thisbée sont définitivement séparés. Strauss reprend le motif de la fête nuptiale de Thésée et d'Hippolyte, mais inversé : le temps a passé, Titania fête ses noces d'argent avec le taureau. Le Minotaure, né de leur union, déplore avec amertume l'absence de la plupart des invités. Seuls sont présents Georg, Helen, Wolf, Helma et Oberon. Un Minotaure élégiaque, raffiné, hypersensible, manifestement amoureux de sa mère, sourd à la présence lascive de la jeune domestique. La défaite d'Oberon et Titania est totale. 
Ces quelques indications sur l'action mouvementée, assez déconcertante et parfois, il faut bien le dire, franchement énigmatique de Der Park permettent de mieux saisir la logique de la pièce, la perspective dans laquelle Botho Strauss cite Shakespeare et la mythologie à la barre du procès qu'il intente à la société où il vit et dont il ressent avec acuité certains déficits. Comme dans Gross und Klein, il cherche à montrer un phénomène de décadence, à stigmatiser, dans les rapports entre les sexes notamment, une dégénérescence de l'instinct de vie, domestiqué par la conscience et le mercantilisme. Les personnages qu'il nous présente sur la scène humaine de la pièce, à l'exception, peut-être, des quatre jeunes gens, ont en commun la perte de la ferveur de vivre, du sentiment poétique de l'existence que l'on sent vibrer dans Le Songe d'une nuit d'été et cette altération est surtout sensible chez les personnages masculins. Le ternissement du désir, de l'Eros qu'Oberon et Titania tentent de faire sourdre à nouveau, doit être compris au sens large : il signifie la disparition d'un élan vital, chez les couples qui organisent leurs liaisons au lieu de les vivre ou qui comblent, comme le font Erstling et Höfling, cette absence par un bavardage anesthésiant. Shakespeare et Le Songe d'une nuit d'été, pièce à la gloire de la poésie, de l'ardeur amoureuse et aussi de l'imagination, fournissent à Strauss le contraste idéal pour faire ressortir l'appauvrissement qu'il ressent. Der Park est la mise en scène d'une contagion par l'inertie, d'une victoire du froid. Cette contagion irréversible et lente, l'effacement de l'image des dieux, constituent le moteur dramatique de l'œuvre qu'il ne faut pas chercher, comme le font certains critiques, dans une évolution des personnages humains qui, même s'ils traversent une crise, ne se transforment pas véritablement mais révèlent plutôt les tendances profondes de leur personnalité. Peter Stein a bien perçu le mouvement de ce voyage au bout de la solitude et de la tiédeur, ciment de cette pièce particulièrement prolixe et touffue, et il a voulu en faire un des axes de son spectacle.

\section{La mise en scène de Peter Stein}

Il est certain que l'intimité intellectuelle de Botho Strauss et de la Schaubühne rend cette mise en scène particulièrement intéressante. Nulle autre troupe n'était aussi qualifiée pour monter Der Park, pour en saisir d'emblée les intentions profondes et leur donner une image scénique convaincante.

La perspective de Stein épouse parfaitement la démarche de l'auteur : il va montrer un songe qui vire au cauchemar, un processus sans retour de dégradation, de désintégration, mot-clé pour le théâtre de Botho Strauss analyste de la dislocation des liens garantissant traditionnellement la cohésion de l'individu et celle du groupe social. Désintégration individuelle. Les dieux s'évanouissent, les hommes s'éloignent, s'isolent dans le repli sur soi, le refus de la communication ou de l'impuissance à communiquer, perdent leur visage. Désintégration du couple, métaphore de l'unité. Image d'un corps social fissuré, dominé par une violence souterraine. Ce processus va rythmer le spectacle de Peter Stein, vaste mise en scène d'une chute généralisée, à l'image de celle de l'homme qui, aux yeux de Strauss, a cessé d'être lui-même, chute symbolisée par un parc sali, témoin d'une nature aliénée, dégradée par la civilisation industrielle et qu'annonce symboliquement, tout au début de la pièce, la chute de Helen au trapèze. Comment Peter Stein procède-t-il pour rendre sensible cette chute aux multiples visages : celle des dieux, celle des hommes, mais aussi celle de l'art et celle du langage? 
Le texte des commentaires du metteur en scène, au moment de la lecture collective avec la troupe, au début des répétitions, constitue une source précieuse de renseignements. La réception du spectacle par la critique théâtrale, particulièrement abondante, apporte également un éclairage intéressant.

\section{Les dieux}

7 Avant de souligner l'extinction d'Oberon et de Titania dans l'anonymat et l'impersonnalité, Peter Stein fait chatoyer l'image de leur transcendance. Il souligne avec brutalité la force de l'érotisme, l'exigence de passion. Bruno Ganz, interprète du rôle d'Oberon et Jutta Lampe, de celui de Titania, dévoilent leur corps nu aux passants et aux spectateurs. Ils se «montrent » comme le rapportent les récits mythologiques et Peter Stein veut ce dévoilement sexuel provocant - des projecteurs éclairent le sexe des deux acteurs - mais également, au-delà de sa crudité, porteur d'une signification symbolique : il doit dire la primauté du désir sur sa réalisation. Sa mise en scène s'emploie à montrer, surtout chez Titania, éternelle amoureuse, toute l'ardeur du désir. La scène où elle implore Cyprian de l'aider à s'accoupler au taureau est, paraît-il, saisissante. Titania, follement éprise du jeune noir; amante obstinée, partie à la recherche d'Oberon après sa disparition. L'intention de Peter Stein est admirablement servie par le talent de Jutta Lampe. Elle parvient, dans ce rôle passablement dangereux, à incarner sur scène, contrairement à un Oberon quelque peu figé dans une attitude solennelle - Stein a voulu le contraste, et le jeu de Bruno Ganz vise la dignité compassée et déclamatoire - une intensité et une grâce extraordinaires, totalement exemptes de mauvais goût, à rendre perceptibles la puissance et la ferveur du désir. Stein suit en cela le texte de Strauss, qui s'inspire lui-même de celui de Shakespeare. Il joue sur l'opposition entre un Oberon autoritaire, engoncé dans son messianisme (et aussi un peu calculateur) et une Titania mobile, plus réaliste que lui, incarnation d'un principe élémentaire de la vie, quête inlassable de l'étranger. Pour magnifier ces instances supérieures "venues d'une autre planète ", Stein souligne également la transcendance par le biais du langage poétique, hérité de Shakespeare dont Strauss cite parfois le texte et le fameux passage où Oberon évoque " le pays où fleurit le thym sauvage ", symbole, dans la pièce, d'un univers poétique aux antipodes de la trivialité du monde moderne. Cette langue poétique, Bruno Ganz en accentue la noblesse pour mieux faire saillir dans la seconde partie, aux actes IV et $\mathrm{V}$, la pauvreté et la standardisation du langage dominant; l'affadissement de l'allure et la platitude de l'expression doivent se rejoindre à la fin du spectacle et donner toute la mesure de la chute du couple royal. En effet, à cette mise en exergue de la divinité, Stein oppose brutalement, aux deux derniers actes, qu'il appelle « la nouvelle pièce " (la pièce " ancienne » est terminée et même périmée, peut-on encore jouer Le Songe d'une nuit d'été ?) la réduction à l'échelle humaine. Bruno Ganz, vieilli de vingt ans, s'est métamorphosé en un personnage jugé effacé et insignifiant. Même image de la domestication chez Titania à l'acte V surtout, mais dans un autre registre : la reine des fées est devenue une grande dame élégante, pleine de mansuétude, donnant une réception dans un salon bourgeois cossu. Ces compositions visent la banalité et l'embourgeoisement. Elles doivent créer un effet de surprise et faire réaliser au spectateur toute l'étendue du rapetissement subi au contact des hommes. 


\section{Les Hommes}

8 La dénaturation des rapports humains et des êtres est à l'image du parc et elle procède d'une dégradation existentielle : à un parc souillé correspondent nécessairement des relations humaines vidées de leur substance.

Les duos de Erstling et Höfling fournissent un bel exemple de cette altération de la communication qui fait que des êtres vivent et parlent ensemble sans se rencontrer véritablement. Stein tient à mettre en évidence l'aspect rituel de leurs discussions, de leurs sempiternelles chamailleries sur des futilités, montrer, en accentuant leur itinéraire en dents de scie, "la fuite totale de la pensée ». Il tient également à faire sentir l'isolement profond du jeune noir qui n'a pas la parole et plus grand chose à voir, pour lui, avec le prince de Shakespeare: c'est un travailleur immigré, plongé brutalement dans une métropole occidentale, obligé de se vendre pour survivre. La mise en scène doit s'efforcer, comme le fait Strauss, de «désorientaliser» le personnage. Mais c'est essentiellement à travers l'image des deux couples, celle des deux hommes plus particulièrement, que Stein s'attache à rendre sensible l'assoupissement de la vie, thème central de la pièce. Le décor et les costumes signalent le confort : appartement design, vêtements à la mode. L'allure et le comportement trahissent le refus des conflits, la fuite devant le risque. Chez Wolf: il a oublié la révolution française et tout l'écœure finalement, comme le lui reproche Helma. Georg organise son ménage à trois parce qu'il veut cumuler tous les conforts, celui de l'amitié et celui de la vie conjugale. Stein souligne cette connivence des deux hommes dans la tiédeur, leur tendance à la compromission; à la fin du troisième acte, ils assistent tranquillement, assis par terre, à l'empoignade des deux femmes. Il veut rendre perceptible une certaine attitude alliant la mollesse du sentiment à une tendance compensatrice à la ratiocination. Les deux femmes du quatuor sont moins engluées dans cette tiédeur. Le personnage de Helma est le plus clair. Elle incarne aux yeux de Stein la représentante typique de la femme qui se définit en fonction de son mari, mais il tient aussi à dire qu'on ne peut pas la réduire à une image trop courte d'épouse cantonnée dans la sphère domestique. Helma-Daphné se transforme en arbre pour échapper aux assauts amoureux de Georg-Apollon. Elle tente de réveiller Wolf et d'éviter l'engourdissement de leur couple dans la vie quotidienne. Elle rejoint Titania dans la mesure où elle incarne un élément stimulant, facteur de cohésion. Helen est plus complexe. Contrairement à Helma, "arbre de vie » au troisième acte, elle suit une route nettement plus dangereuse qui la conduit vers la solitude et la pousse dans les bras de la mort. C'est sur elle que la magie d'Oberon aura l'effet le plus brutal. La composition de Libgart Schwarz, interprète du rôle, révèle la direction prise par Peter Stein, désireux de faire apparaître en elle tout le « sang noir » qui coule dans les veines de la société allemande. L'actrice campe avec véhémence un personnage violemment réactionnaire et irrationnel. Elle est la sorcière qui mène le sabbat de la nuit de la SaintJean, où Peter Stein place le sommet dramatique du spectacle, prélude au calme inquiétant des deux derniers actes. A ce quatuor, où les femmes jouent une partie nettement plus dynamique que les hommes, s'oppose celui des jeunes gens que Stein a voulu être à la fois les témoins d'un profond désarroi mais aussi d'une révolte et d'une énergie positives. Le personnage de la jeune fille exprime la désorientation d'une jeunesse récusant l'égoïsme des adultes. Stein insiste sur le fait que ses paroles ne correspondent pas à un discours analytique, instrument d'une prise de conscience, mais qu'elle articule inconsciemment les thèmes qui obsèdent les jeunes de son âge : 
recherche de l'identité, d'autres formes de vie, avantages et inconvénients de la solitude, un mot qui revient constamment dans sa bouche. Stein veut aussi indiquer que ces jeunes constituent une bande toujours en maraude dans le parc, à l'affût d'une victime mais aussi d'une expérience neuve, à suggérer un va-et-vient entre l'agressivité délibérée à l'égard des adultes dont ils exploitent les moindres faiblesses (Oberon / Mittentzwei va en faire les frais dans ce que Stein appelle « un show de la destruction du père ») et la recherche confuse d'une issue, le rêve d'une autre vie. Ils sont pour lui, dans la perspective mythologique de la pièce, "les démons inférieurs", " des sorcières en miniature, sorties des toilettes du Tiergarten». Il font partie intégrante de la fantasmagorie, représentants d'un comportement antinomique de celui des adultes et, à ce titre, les alliés de Titania et d'Oberon, à leurs côtés dans le camp des forces qui œuvrent contre la déliquescence de la vie sociale. Peter Stein a nettement ressenti et exploité cette filiation, que la critique n'a pas toujours très bien perçue.

\section{L'art}

Le personnage de Cyprian contribue fortement à créer l'atmosphère caractéristique de la pièce, mélange intime de mystère, d'irréalité, induits par la présence poétique de Shakespeare et de la mythologie grecque, et de réalisme, alimenté par la chronique de la vie quotidienne. Cyprian - le nom renvoie à l'histoire religieuse, à la passion amoureuse et à la magie - est un créateur, dépositaire de pouvoirs divins. Il trahit son maitre, mais avec le désir de transformer le regard des hommes. Strauss l'associe à la figure mythologique de Dédale, père d'Icare, artiste, architecte, ingénieur. Il construisit, outre le labyrinthe, dont il aida Thésée à s'échapper, des marionnettes pour les enfants de Minos et de Pasiphaé. Peter Stein discerne en lui une image de l'artiste magnifiquement doué, l'auteur de petites sculptures magiques évoquant la poterie Cretoise en terre cuite. C'est aussi à ses yeux, dans la pièce, l'un des tenants du désir et de l'érotisme. Mais il voit également un artiste déchu qui ne peut résister, pour des raisons matérielles, à la commercialisation abusive de son talent, qui se "vend " à son tour, en frôlant la charlatanerie et dévalue du même coup son œuvre et « l'esprit de la nature » qu'il transforme en " marchandise de masse ».

Selon Stein, l'interprète du rôle ne doit pas chercher à résoudre la contradiction, à justifier ou expliquer Cyprian, mais au contraire, par une composition très contrastée à faire cohabiter abruptement les deux versants du personnage, le côté Puck et le mercantilisme, à montrer un Puck inventif mais qui tourne mal. Cyprian s'inscrit dans le processus de dégradation généralisée: avec sa fin minable dans le parc, son affairisme, ses entreprises qui avortent, sa magie impuissante à sauver les hommes. La faillite de Cyprian veut-elle exprimer celle de l'Art, devenu incapable pour Strauss de transcender la vie humaine, capitulation qui serait le corollaire de celle d'Oberon? Cyprian est un personnage mystérieux et névralgique dont une mise en scène ne doit pas sous-estimer l'importance. Peter Stein le met au premier plan de la sienne, parfaitement servi par W. Schmidinger, interprète du rôle.

\section{Le langage}

Comme Kroetz ou Handke, Strauss est l'héritier de Horvàth dont le théâtre s'emploie à faire émerger la vérité profonde des êtres à «démasquer la conscience » par la 
médiation du langage. Ses personnages - le phénomène est particulièrement net dans Gross und Klein ou Kalldewey, Farce - se repèrent d'abord par une manière de s'exprimer, un vocabulaire, une syntaxe révélateurs de leur personnalité mais aussi du poids de leur environnement. Dans Der Park, Strauss utilise méthodiquement le procédé de la citation contrastive de plusieurs niveaux de langue destinés à se heurter entre eux et à imprimer un certain rythme au spectacle. Il joue sur plusieurs registres : il utilise naturellement la langue noble et poétique de Shakespeare, mais elle n'est pas l'apanage de Titania et d'Oberon; il introduit parfois un brusque décrochage dans le mode d'expression d'un personnage, générateur d'une dramatisation supplémentaire. Il cite le langage de la "Szene", de la "Beziehungkiste», les jargons du monde moderne, la langue de bois des affaires, des médias, de la publicité, celle des jeunes gens du parc. Stein a tenu à exploiter ce panachage des styles et à montrer que l'abdication de la personne avait pour écho privilégié la standardisation, l'amenuisement de la parole. La scène du constat après l'accident (très symbolique!) qui oppose Oberon / Mittentzwei à Wolf et Helma, est un modèle du genre. Une mise en scène soucieuse de rendre justice à la pièce ne peut laisser dans l'ombre ce jeu de variations subtiles qui l'articulent. Peter Stein y a été très sensible car c'est à ses yeux un des ressorts de la poésie et du charme de la pièce ; il a surtout joué sur la confrontation des deux langages antagonistes qui démarquent respectivement le territoire du mythe, de la vie originelle, et celui de la vie quotidienne à notre époque. La mise en scène de la scène II de l'acte III utilise systématiquement ce choc des deux langages: l'affrontement des êtres se double d'un affrontement des styles.

Dans le même ordre d'esprit, Stein veut aussi suggérer l'écho du langage collectif à travers l'expression individuelle. La voix de Helen fait entendre le discours de la droite néo-nazie. Höfling et Erstling signalent le verbiage décousu des médias. Les jeunes gens parlent le langage de la "Szene» ouest-allemande que Strauss connaît bien. Le mouvement de la pièce, à cet égard, est très net : la langue de Shakespeare s'estompe pour faire place aux différents "prêt-à-parler » en usage aujourd'hui. Le monologue final du Minotaure, évoquant une dernière fois l'harmonie et la paix d'un âge d'or où l'homme et la nature seraient réconciliés, est une ultime résurgence du langage poétique du Songe (devenu avec Botho Strauss le mensonge) d'une nuit d'été.

\section{La réception par la critique de presse}

Comment la critique théâtrale a-t-elle reçu la mise en scène de Stein? Les jugements franchement hostiles sont rares et ils mettent surtout en cause la pièce elle-même. Quelques restrictions cependant: on reproche à Stein d'avoir été trop didactique dans sa démarche, trop étroitement fidèle à Strauss, de n'avoir pas su résister à certains effets de théâtre gratuits. Mais les critiques, dans leur grande majorité, sont séduits par un spectacle qu'ils jugent certes long mais fascinant... Le mot revient fréquemment dans les commentaires. Fascinant par son audace et sa richesse scénique. Ils lui font en effet deux compliments de taille. La mise en scène ne s'est pas dérobée devant l'ambition intellectuelle de l'œuvre; elle a voulu montrer, comme Strauss, l'agonie des mythes et des utopies, le chaos latent qui sommeille derrière l'ordre d'une société guettée par la mort de la communication humaine et elle a su éviter l'écueil du désamorçage dans la comédie de mœurs sur fond de fantasmagorie à caractère décoratif. Et puis, surtout, elle a su faire la part belle au théâtre. Presque tous les 
critiques s'accordent à souligner le plaisir ressenti devant les qualités visuelles du spectacle, la puissance de suggestion de certains tableaux, la rapidité du rythme dans l'enchaînement des différentes scènes, la force inventive du décor de Karl-Ernst Herrmann, servi par les puissants moyens techniques de la nouvelle Schaubühne. Grâce aux vertus d'une théâtralité qui a vu et senti juste, la pièce de Botho Strauss, parfois menacée par sa richesse et son abondance, retrouve sur la scène de la Schaubühne toute sa cohésion et donc sa force théâtrale.

La réussite de Peter Stein est incontestable, mais on peut se demander si des metteurs en scène moins au fait de la pensée de Botho Strauss, dirigeant des troupes moins brillantes, ne risquent pas de perdre dans les allées parfois tortueuses de Der Park qui n'est certainement pas une œuvre facile à maîtriser. Ils risquent en effet de la tirer trop lourdement vers le théâtre de boulevard, ou même vers la revue de cabaret : il y a dans la pièce de nombreux numéros assez spectaculaires, susceptibles d'être montés en épingle pour plaire au public, aux dépens de la cohésion de la pièce. Ils risquent aussi de succomber à l'exotisme de la composante mythologique, de refuser l'amertume de la critique sociale pour faire chatoyer la féerie que Strauss s'applique à détruire froidement, de vouloir à tout prix éviter le naufrage du Songe d'une nuit d'été. Der Park est une pièce noire. Comme dans le drame joué à la fin de Hamlet, où le meurtre du père est révélé publiquement par la fiction du jeu théâtral, l'artificialité du spectacle dénonce dans cette œuvre la mort du désir. Combien de scènes de théâtre sauront-elles exprimer dans un langage direct et fort l'analyse subtile, confinant parfois au maniérisme mais peut-être lucide de Botho Strauss?

\section{NOTES}

1. Ce texte a été publié dans Cahiers d'Etudes Germaniques, $\left(1985, n^{\circ} 9\right)$. Il est reproduit avec l'aimable collaboration de l'auteur.

\section{AUTEURS}

\section{GÉRARD JUGAN}

Gérard Jugan est maître de conférences de littérature et civilisation allemande à l'Université de Franche-Comté. Il a publié un dossier sur Büchner dans Coulisses $n^{\circ} 7$. 